TITLE:

\title{
Molecular composition of films and solid particles polymerized in fluorocarbon plasmas
}

$\operatorname{AUTHOR}(\mathrm{S})$ :

Takahashi, Kazuo; Tachibana, Kunihide

\section{CITATION:}

Takahashi, Kazuo ...[et al]. Molecular composition of films and solid particles polymerized in fluorocarbon plasmas. JOURNAL OF APPLIED PHYSICS 2001, 89(2): 893-899

\section{ISSUE DATE:}

2001-01-15

URL:

http://hdl.handle.net/2433/51633

\section{RIGHT:}

Copyright 2001 American Institute of Physics. This article may be downloaded for personal use only. Any other use requires prior permission of the author and the American Institute of Physics. 


\title{
Molecular composition of films and solid particles polymerized in fluorocarbon plasmas
}

\author{
Kazuo Takahashi ${ }^{\mathrm{a})}$ and Kunihide Tachibana \\ Department of Electronic Science and Engineering, Kyoto University, Yoshida-Honmachi, \\ Sakyo-ku, Kyoto 606-8501, Japan
}

(Received 22 June 2000; accepted for publication 30 October 2000)

In fluorocarbon $\left(\mathrm{C}_{4} \mathrm{~F}_{8}\right)$ plasmas, formation mechanisms of polymers were investigated by the characterization with x-ray photoelectron spectroscopy (XPS) and gel permeation chromatography (GPC). The molecular compositions of the polymers in the films deposited on the substrate and in the particles formed in the gas phase were elucidated by these chemical analyses. The XPS results showed that the particles were carbon-rich and composed of highly branched molecules in contrast to the film composition. From the GPC measurements, the particles were found to contain ultrahigh mass polymers, whose molecular weights were around 100000 . On the contrary, the deposited film contained polymers with molecular weights distributed below 2000, in which oligomers, monomers, and fragmented products were included. Present study suggests that these polymers are involved in the formation of crosslinked networks of the films and the particles via surface reactions, where the crosslinking is enhanced by the ion bombardment. (C) 2001 American Institute of Physics.

[DOI: $10.1063 / 1.1334636]$

\section{INTRODUCTION}

In recent ultralarge scale integration (ULSI) device manufacturing, fluorocarbon plasmas are applied not only to selective etching of $\mathrm{SiO}_{2}$ over $\mathrm{Si}$ but also to chemical vapor deposition (CVD) of fluorocarbon film as a low-dielectric constant material. ${ }^{1-4}$ Surface processes occurring in these plasmas are common for the formation of fluorocarbon polymer films on the Si surface. Therefore, the understanding of polymer deposition mechanisms and their control are important for these technologies. Although small radicals $\left(\mathrm{CF}_{x}\right.$, $x=1-3)$ and their ions have been measured systematically in the gas phase by spectroscopic and mass spectrometric methods ${ }^{5-9}$ and chemical composition of surface film has been extensively investigated by using x-ray photoelectron spectroscopy (XPS), ${ }^{10}$ responsible surface reactions for the polymer formation are still under discussion. It is due to the complexity of the whole reactions that determine the deposition rate and the chemical composition of the film. The existence of intermediate precursors as associated molecules have also been suggested, ${ }^{11-13}$ which are produced by polymerization reactions in the gas phase. For their measurement we have developed electron attachment mass spectrometry (EAMS), ${ }^{14-16}$ and showed the presence of a fairly large amount of larger molecular weight species in various fluorocarbon plasmas. However, the method is limited to species with the mass number of less than 1000 or so.

Since it is still suspected that further polymerized species are contributing to the deposition with a higher sticking probability, we try to apply gel permeation chromatography (GPC) in this work for the measurement of species with molecular weights of up to $10^{7} \cdot{ }^{17}$ Production of the polymerized species may occur not only in the gas phase but also on the

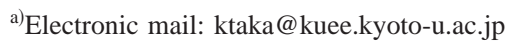

surface. Therefore, characterization by GPC is done for the particles produced in the gas phase as well as the deposited films on the substrate. The chemical compositions of films and particles are analyzed by XPS. From these experimental results the polymerization mechanisms and the contribution of these polymers to the film deposition and the particle formation are discussed.

\section{EXPERIMENTAL METHODS}

In the present experiments, octafluorocycrobutane $\left(c-\mathrm{C}_{4} \mathrm{~F}_{8}\right)$ was used as a kind of fluorocarbon compound. The gas was supplied by a mass flow controller from an upper shower electrode into a parallel plate reactor as shown in Fig. 1. The gas flow rate was 1.7 standard $\mathrm{cm}^{3}$ per minute $(\mathrm{sccm})$. The plasma reactor was evacuated by a turbomolecular pump (TMP) and a rotary vane pump (RP). For the plasma generation, a rf (13.56 MHz) power supply was connected to the upper electrode, of which the power density was maintained at $0.15 \mathrm{~W} / \mathrm{cm}^{2}$. The gas pressure in the chamber was varied between 23 and 250 mTorr.

A Si wafer was set on the lower electrode after all impurities were rinsed out by ethanol and acetone. Then, the substrate was exposed to the plasma for $15 \mathrm{~min}$ by suspending it at a floating potential. In addition to this typical treatment, some substrates were treated on the powered electrode under the influence of the self-bias voltage $\left(V_{\mathrm{dc}}\right)$ by changing the rf power connection to the lower electrode. The particle sample was prepared as follows; after the plasma treatment particles dropped on a Si substrate were raked up with a stainless steel spatula. Although it was difficult to avoid completely the inclusion of film flakes caused by the spatula, we tried to minimize the effect by a careful handling.

The molecular weight distribution of the products contained in the film sample and the particle sample was ana- 


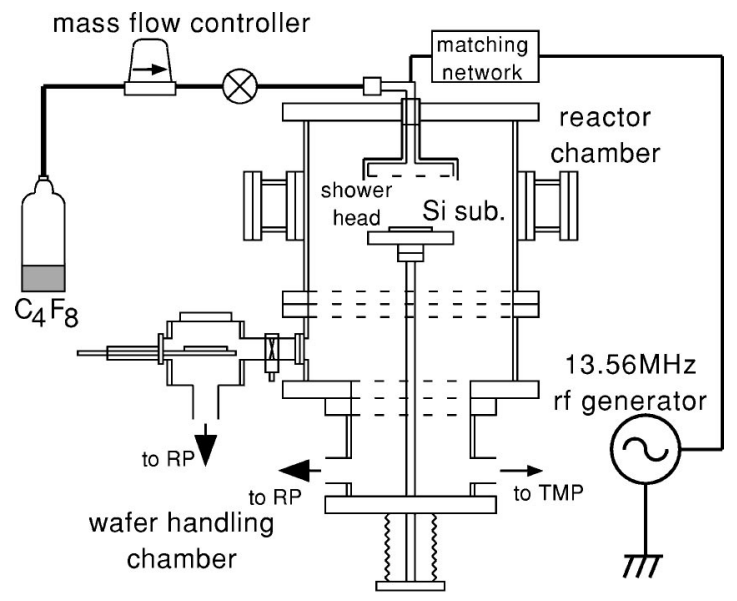

FIG. 1. Schematic of the experimental setup.

lyzed by GPC. For the GPC measurement, these samples were dipped in a small amount of tetrahydrofuran (THF) solvent for $5 \mathrm{~min}$ at room temperature, and soluble components were extracted from the samples. Since a carbon atom and a fluorine atom form strong chemical bonds; the bond energies of $\mathrm{C}-\mathrm{F}, \mathrm{C}-\mathrm{C}$, and $\mathrm{C}=\mathrm{C}$ are 83, 116, and $146 \mathrm{kcal} /$ mol, respectively, ${ }^{18}$ we expect that the fluorocarbon polymers can be extracted from the film and particle samples without fragmentation of the molecular structures in the treatment. No chemical reaction occurs between the extracted molecules without radical sites and the THF solvent, since the chemical composition of polymerized fluorocarbon molecules is similar to polytetrafluoroethylene (PTFE) being known as a chemically stable substance to the solvent. However, free radicals can be included in plasma polymerized polymer and may lead some chemical reactions. One free radical will be involved in a few plasma polymerized molecules. ${ }^{19}$ In such a large molecule detected in the GPC measurement, the radical site can be ignored for determining molecular weight distribution of the polymerized fluorocarbon molecule. ${ }^{20}$ The prepared sample in the THF solution was injected into the GPC column after a filtration process to remove insoluble substances. The column separated the composites of the sample into several groups depending on their molecular sizes. The fluorocarbon molecules passed through the column were detected by two methods based on the differential refractive index (RI) and the absorption of ultraviolet (UV) light. To determine the wavelength of UV light, the optical property of a THF solution held in a silicate glass tube was measured with a spectrophotometer from UV to visible regions. The absorption spectrum of fluorocarbon molecules showed a broad increasing peak toward the shorter wavelength region below $600 \mathrm{~nm}$ as shown in Fig. 2. From this result, the wavelength for the GPC measurements was set at $340 \mathrm{~nm}$, where both the absorption and the UV intensity were at reasonable levels. The relation between the retention time of a fluorocarbon molecule in the column and the molecular weight was determined by using a polystyrene standard sample. Although the retention time may depend on molecular shape such as linear and branched chain, we were

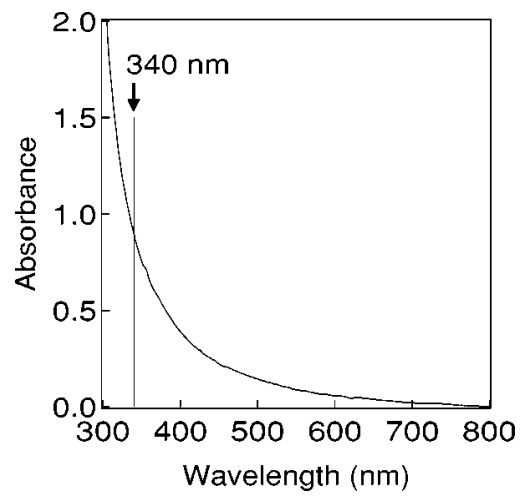

FIG. 2. Absorption spectrum of fluorocarbon molecules extracted by THF treatment.

not hindered from discussing molecular weight distribution of the fluorocarbon molecule qualitatively.

On the other hand, the insoluble components of the film and particle samples were characterized by XPS for the chemical bond compositions. The XPS spectra were taken by using the $\operatorname{Mg} K \alpha$-line with an acceleration energy of $13 \mathrm{keV}$.

\section{RESULTS}

In the whole tested gas pressure range, amorphous fluorocarbon film was deposited irrespective of the substrate condition. Solid particles, however, were observed only at the pressure higher than $50 \mathrm{mTorr}$, whose diameter ranged between 0.5 and $2.3 \mu \mathrm{m} .{ }^{21}$ The particle production was enhanced and the agglomerates such as shown in Fig. 3 appeared when the pressure was increased. The agglomerates were composed by many particles of a few micrometer in their diameter.

Figures 4 and 5 show GPC curves of extracted species detected by the UV and RI methods, respectively. The notations of "Film-L" and "Film-H" indicate the signals of the film samples prepared in the condition at low pressure of 23 mTorr and at high pressure of 250 mTorr, respectively. "Particle-H" indicates the GPC curve of the extract from particles produced in the same condition as in the case of Film-H. The responsibilities of the UV and RI signals in the measurements depend not only on the density of each fluo-

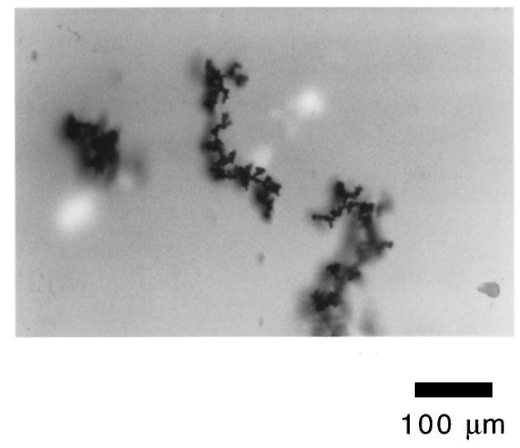

FIG. 3. Optical microscope image (differential interference contrast) of surface morphology of $\mathrm{Si}$ substrate exposed to $\mathrm{C}_{4} \mathrm{~F}_{8}$ plasma for 15 min under the condition of $1.7 \mathrm{sccm}, 200 \mathrm{mTorr}$, and $0.15 \mathrm{~W} / \mathrm{cm}^{2}$, showing the presence of agglomerates of particles. 

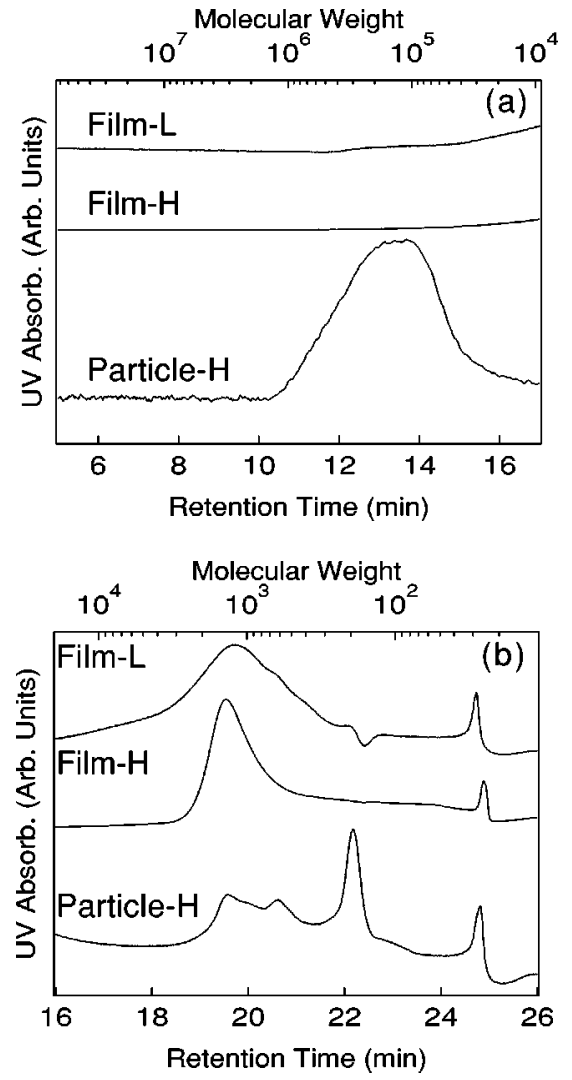

FIG. 4. GPC curves of THF extracts from three samples measured by UV transmittance method at two retention periods: (a) from 5 to $17 \mathrm{~min}$ and (b) later than 16 min. Notations "Film-L," "Film-H," and "Particle-H" correspond to film and particle samples prepared at low pressure of $23 \mathrm{mTorr}$ (indicated with L) and high pressure of $250 \mathrm{mTorr}(\mathrm{H})$.

rocarbon molecule in the solution but also on its specific efficiency. Thus, the appearance of molecular weight distribution measured by the UV absorption method is not necessarily the same as that measured by the RI method even in the measurement of the same sample. Therefore, we used both methods complementarily to estimate the possible molecular weight distribution of polymers.

\section{A. Structure of surface film}

According to the GPC curves of Film-L and Film-H shown in Figs. 4 and 5, it is seen that the extracted components of the deposited films do not depend on the pressure condition. The molecular weight distribution extends up to 2000 in the UV measurement. In the RI measurement, however, the signal around 2000 was small while a sharp peak appeared around 300. The reason of this different appearance between UV and RI methods is not clear, but may be attributed to the fact that the difference in refractive indexes of fluorocarbon polymers and THF is very small. (The refractive indexes of both THF and hexafluorobenzene as a reference of polymer molecules are around 1.4 as measured by using D lines of $\mathrm{Na}$ around $589 \mathrm{~nm}^{22}$ ) Although we could not estimate the amount of molecule corresponding to each peak in GPC curves since concentration of solution was impossible to be measured, it is true that the molecular weight has a broad distribution from 100 to 2000 . This indicates that
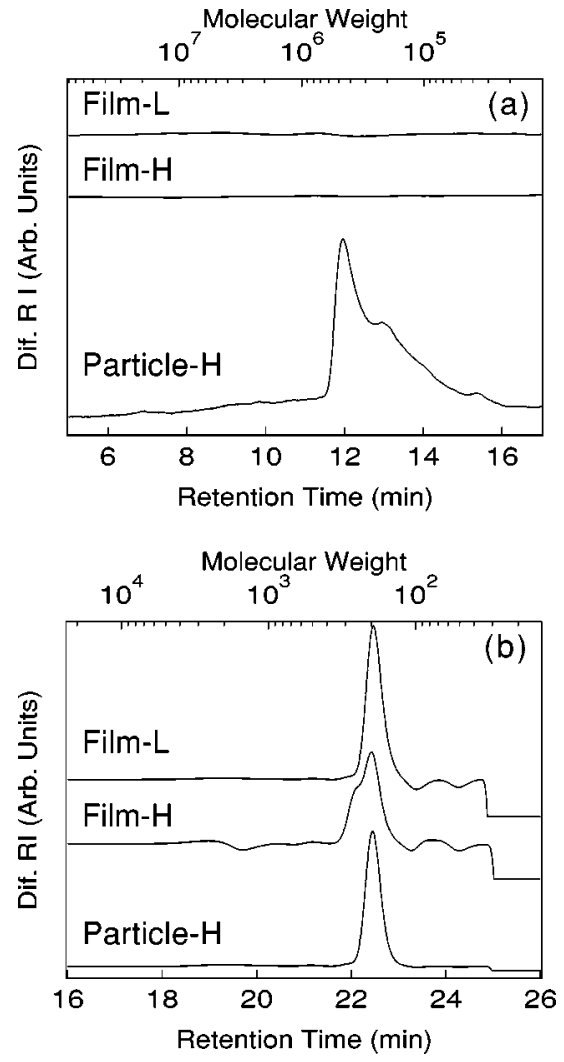

FIG. 5. GPC curves for the same three samples as in Fig. 4 measured by RI method.

the plasma polymerized film contains oligomers, monomers, and other reaction products with relatively small molecular weights.

In the THF treatment, it turned out that the film was composed of not only the soluble substance but also the insoluble substance. The THF solubilities of the films prepared at a low pressure condition of $23 \mathrm{mTorr}$ and a high pressure of 250 mTorr were $33 \%$ and $75 \%$ in their weights, respectively. Generally, the interaction between a $\mathrm{Si}$ substrate and a fluorocarbon molecule by the van der Waals force is proportional to the size of the molecule. For a larger fluorocarbon molecule the interaction tends to exceed the solvability in THF, and it remains on the substrate as the residue probably in the form of a porous film. Besides, there may be any difference in the chemical bond composition between the soluble substance and the insoluble residue. Therefore, the XPS analysis was employed to compare the compositions of the as-deposited film and the residue after the THF washing.

The XPS spectra of $\mathrm{C}_{1 \mathrm{~s}}$ signal from the film prepared at 23 and 250 mTorr are shown in Figs. 6 and 7, respectively, where (a) and (b) indicate the spectra of as-deposited film and that of washed one, respectively. The spectra were deconvoluted into five chemical bond components; $\mathrm{C}-\mathrm{C}$ at $285.0 \mathrm{eV}, \mathrm{C}-\mathrm{CF}_{x}$ at $287.3 \mathrm{eV}, \mathrm{CF}$ at $289.5 \mathrm{eV}, \mathrm{CF}_{2}$ at 292.1 $\mathrm{eV}$, and $\mathrm{CF}_{3}$ at $294.0 \mathrm{eV} .^{23}$ The composition ratio of these bonds was estimated as shown in Fig. 8, where each component was assumed to have a Gaussian distribution. Table I lists the overall atomic ratio of fluorine to carbon, F/C, derived from the XPS analysis. The ratio did not depend on the 

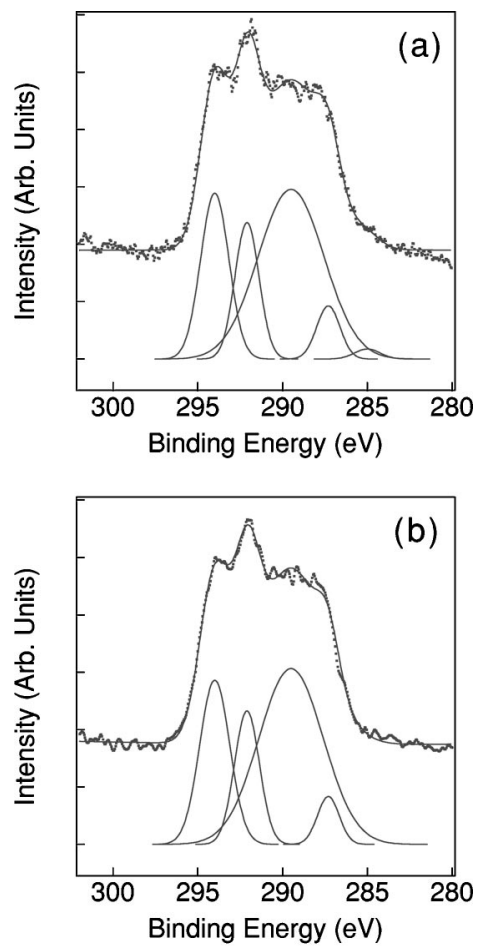

FIG. 6. XPS spectra of $\mathrm{C}_{1 s}$ measured for a film sample prepared at low pressure (23 mTorr) condition (dotted curve): (a) as-deposited film and (b) residue after THF treatment. Solid curve indicates the fitted result with five Gaussian peaks for chemical bond components corresponding to $\mathrm{C}-\mathrm{C}$ at $285.0 \mathrm{eV}, \mathrm{C}-\mathrm{CF}_{x}$ at $287.3 \mathrm{eV}, \mathrm{CF}$ at $289.5 \mathrm{eV}, \mathrm{CF}_{2}$ at $292.1 \mathrm{eV}$, and $\mathrm{CF}_{3}$ at $294.0 \mathrm{eV}$.

THF treatment so much for the films prepared in both low and high pressure conditions. However, the chemical bond composition of the film prepared at a high pressure of 250 mTorr showed a noticeable change after the THF treatment. (For the film prepared at a low pressure of 23 mTorr, there was almost no change after the THF treatment, since the solubility of the film in THF was small.) It was noted that the THF treatment washed out the $\mathrm{CF}_{3}$ bond component and exposed the CF bond component as seen in Figs. 7(a), 7(b), and $8(\mathrm{~b})$. Since the size of a molecular unit composing the crosslinked film is large and insoluble in the THF treatment, it is imagined that the remaining part has a highly crosslinked structure with much abundant CF bonds. Large molecules are produced possibly by the reactions on the surface rather than the reactions in the gas phase, because such large molecules should form solid particles if they are produced in the gas phase. On the other hand, the soluble substance may be regarded as the reaction products in the gas phase.

This change in the spectrum by the THF treatment indicates the difference in chemical compositions between the surface product and the gas phase product. The insoluble residue in the film includes more $\mathrm{CF}$ bonds than $\mathrm{CF}_{3}$ bonds due to its crosslinked network, while the substance extracted by the THF solvent includes more $\mathrm{CF}_{3}$ bonds. Thus, it is suggested that the species produced in the gas phase contain more $\mathrm{CF}_{3}$ bonds and, therefore, have shorter molecular chains with the molecular weights below 2000 .
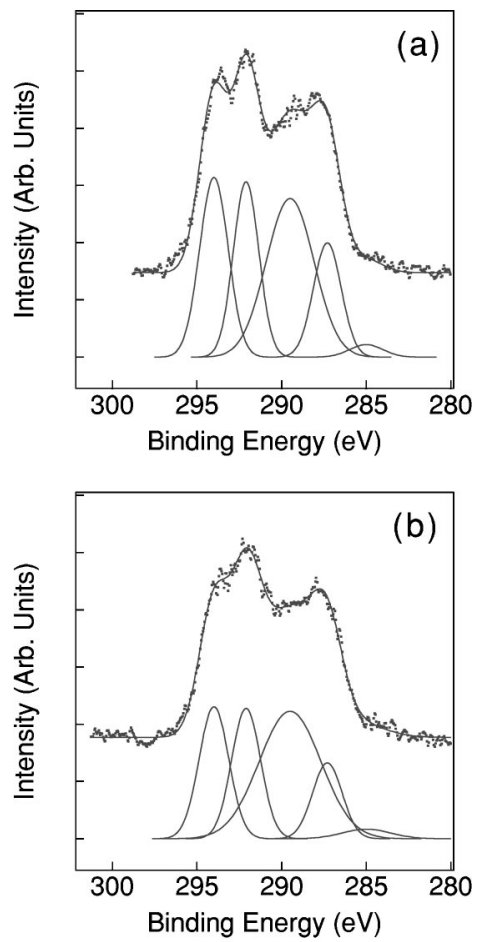

FIG. 7. XPS spectra of $\mathrm{C}_{1 s}$ measured for a film sample prepared at high pressure (250 mTorr) condition (dotted curve): (a) as-deposited film and (b) residue after THF treatment. Solid curve shows the fitted result as in Fig. 6.

In order to see the effect of ion bombardment to the polymerization, some samples were prepared on the powered electrode by changing the rf power density from 0.079 to $0.71 \mathrm{~W} / \mathrm{cm}^{2}$. The THF solubility of these films is shown in
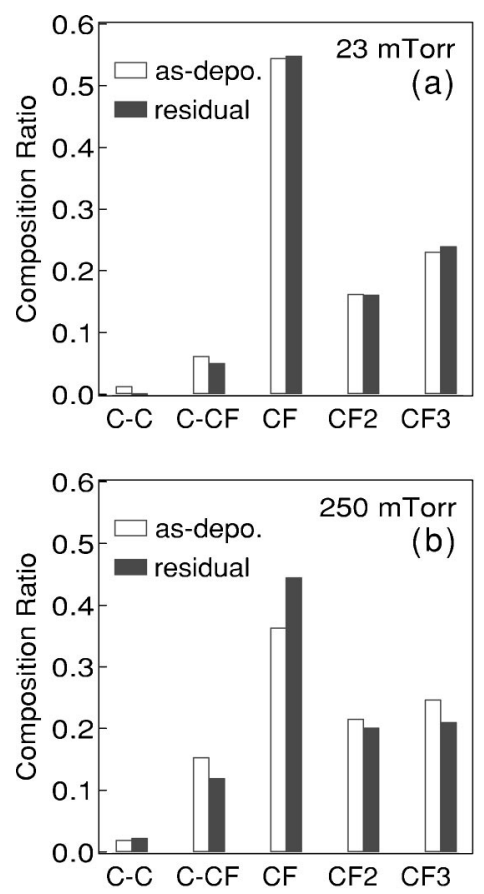

FIG. 8. Comparison of chemical bond composition between as-deposited film and residue after THF treatment derived from XPS analysis for samples prepared at (a) low pressure (23 mTorr) and (b) high pressure (250 mTorr) conditions. 
TABLE I. The atomic ratio of fluorine to carbon, F/C, in fluorocarbon films.

\begin{tabular}{ccc}
\hline \hline Pressure (mTorr) & as-deposited & THF treated \\
\hline 23 & 1.28 & 1.37 \\
250 & 1.29 & 1.25 \\
\hline \hline
\end{tabular}

Fig. 9. The absolute value of self-bias voltage, $\left|V_{\mathrm{dc}}\right|$, increased from 4 to $27 \mathrm{~V}$ with the rf power, and the solubility decreased with the increase of $\left|V_{\mathrm{dc}}\right|$. This result clearly shows that the crosslinking reactions within the deposited film are enhanced on the surface by increasing the bombarding ion energy, indicating that the kinetic energy deposited by the ion bombardment can contribute to the polymer network formation.

\section{B. Structure of gas phase particle}

From the GPC results shown in Figs. 4 and 5, it became clear that the particles formed in gas phase contained polymers of ultralarge molecular weights around 100000 . In addition, species with molecular weights less than 2000 were also observed in the extract from the particles as in the case of the deposited film. In order to explain the difference in the molecular weight distribution between the film and particle samples found in the GPC measurements, the chemical bond composition of those samples was measured by XPS.

In the first measurement, the XPS spectrum of the particles was almost the same as that of the film, because the particles were possibly covered with the fluorocarbon films after dropping on the wafer. ${ }^{21}$ In order to remove the films on the particles, Ar ion sputtering was performed in the XPS system using a low-energy ion beam so as to avoid the transformation of the chemical composition. The XPS spectrum of the particles so obtained is obviously different from that of the film as shown in Fig. 10. The chemical bond compositions also show a clear distinction between them (Fig. 11); the content of $\mathrm{C}-\mathrm{CF}_{x}$ bond in the particles was more than that in the film. This result indicates that the branching of carbon network was promoted in the precursor molecules composing the particles. This is also consistent with the GPC result that the particles are composed of the ultralarge molecular weight polymers. On the other hand, the atomic ratio

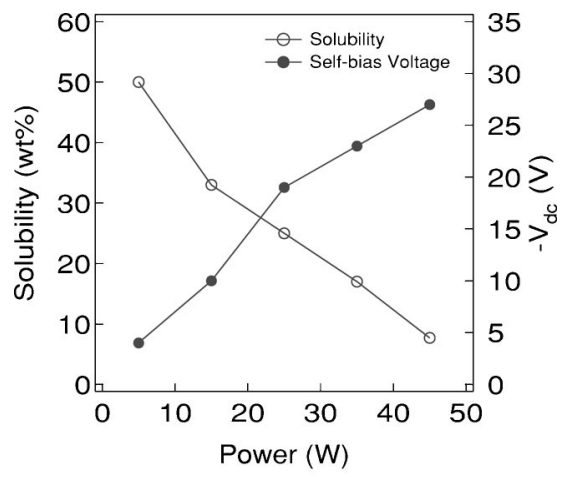

FIG. 9. THF solubility (wt \%) of films prepared on powered electrode plotted as a function of rf power. Self-bias voltage appearing at the electrode is shown on the right vertical axis.

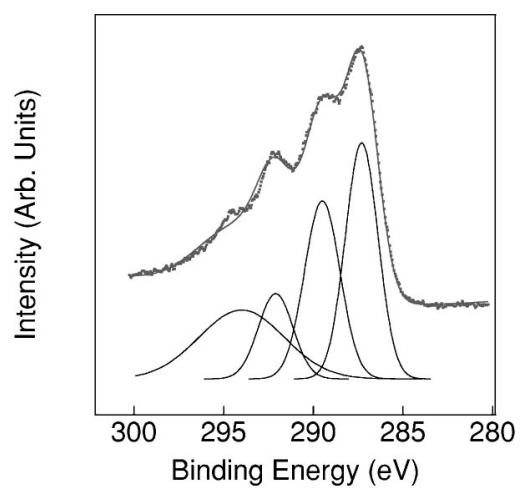

FIG. 10. XPS spectrum of a particle sample (dotted curve). Solid curve shows the fitted result as in Fig. 6.

of fluorine to carbon, $\mathrm{F} / \mathrm{C}$, in the particles was 1.13 , which was smaller than all values listed in Table I. This result of carbon-rich content in the particles also shows that they are composed of crosslinked large molecules. Since a $\mathrm{C}-\mathrm{F}$ bond is weaker than a $\mathrm{C}-\mathrm{C}$ bond, the result also implies the possibility that the gas phase reaction progresses with taking off fluorine atoms.

\section{DISCUSSION}

The film structure investigated by the THF treatments enables us to elucidate a part of the polymerization processes in fluorocarbon plasmas. In gas phase reactions, the primary molecules as the film precursors are produced, whose molecular weights are distributed below 2000. These primary molecules may result from the polymerization between smaller molecules of the size of parent molecules, since the film was found to include much content of the species with molecule weights of around 200 [Fig. 5(b)]. The vapor pressure of the primary molecule may be extremely low, since melting point of a substance composed of such large molecule exceeds $100^{\circ} \mathrm{C}$, e.g., melting point of perfluorotetracosane $\left(\mathrm{C}_{24} \mathrm{~F}_{50}\right)$ is around $190^{\circ} \mathrm{C}$. Therefore, one can expect that such molecules will be adsorbed on the surface immediately after production of the molecule. When the primary molecules reach to the surface, the polymerization reaction is initiated between them and the crosslinked network is constructed to result in the formation of polymer film. The surface reaction of the adsorbates must be induced or enhanced

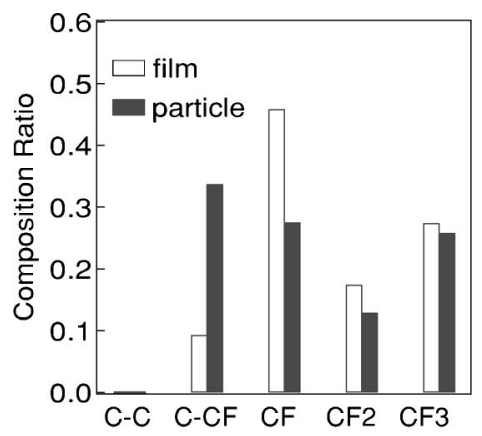

FIG. 11. Comparison of chemical bond composition between as-deposited film and particle samples prepared at the same high pressure $(250 \mathrm{mTorr})$ condition. 


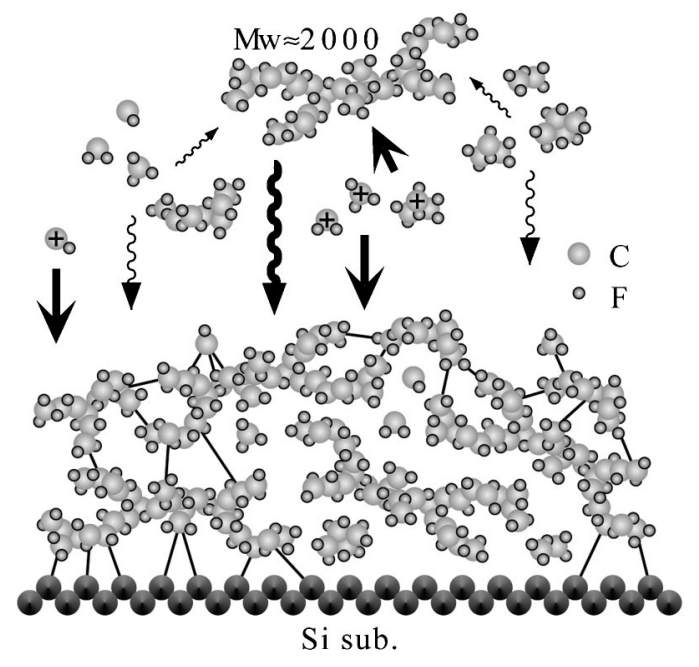

FIG. 12. Conceptual illustration of film structure formed on $\mathrm{Si}$ substrate. ( $M_{w}$ is molecular weight of polymers estimated from GPC measurement.)

by activating them with the irradiation of ions and radicals from the plasma. In addition to their chemical potentials, the kinetic energy released by the ion irradiation can also contribute to the reaction. Thus, we suggest a model for the film formation on the surface as shown schematically in Fig. 12. First, primary molecules are produced by mutual reactions of radicals, ions, and parent molecules in the gas phase. Then, these primary molecules stick on the surface. These molecules associate each other with the help of the ion and radical bombardments. Finally, the crosslinked network is formed, but a part of the primary molecules is left unlinked.

On the other hand, the particles formed in the gas phase are composed of the ultralarge polymers with molecular weights of around 100000 . This fact indicates that such polymers are produced while they are suspended in gas phase and become the precursor of the particle formation. In the plasma, an electron can attack a carbon atom or its bond at a random site in a large molecule and produce a radical site. Then, the radical site works as a polymerization site for the crosslinking of the molecule in the gas phase. The larger a molecule is, the more radical sites for the polymerization are formed in the molecule. As a result of the series of polymerization reactions, a large molecule tends to form a cluster. The cluster can be charged negatively due to the difference in the mobility between electrons and ions in the plasma. The charged clusters thus formed are trapped and accumulated in the plasma by the balance of various forces acting on them: electrostatic force, gravity, ion drag force, etc. ${ }^{24}$

Here, we try to evaluate the geometrical dimension of a polymer chain with a molecular weight of 100000 . Unperturbed molecular dimension is estimated in the simplest model for a linear chain. ${ }^{25}$ In this calculation, it is assumed that the $\mathrm{C}-\mathrm{C}-\mathrm{C}$ bond angle and length in a fluorinated carbon structure are the same as in a hydrogenated carbon structure and the shape of the whole molecular chain is determined by the successive combination of $\mathrm{C}-\mathrm{C}-\mathrm{C}$ units. The root-mean-square end-to-end distance of a free rotational chain is given by the following equation: ${ }^{25}$

$$
\overline{r_{0}^{2}}=n a^{2} \frac{1+\cos \theta}{1-\cos \theta}-2 n a^{2},
$$

where $n, a$, and $\theta$ are the number of $\mathrm{CF}_{2}$ as the smallest unit of the fluorocarbon chain, the bond length of $\mathrm{C}-\mathrm{C}(0.154$ $\mathrm{nm}^{17}$ ) and the bond angle of the $\mathrm{C}-\mathrm{C}-\mathrm{C}$ structure $\left(109.5^{\circ}\right)$, respectively. Because the molecular weight of $\mathrm{CF}_{2}$ is $50, n$ becomes 2000 for a chain with molecular weight of 100000 . Thus, the root-mean-square end-to-end distance of the chain $\left(\overline{r_{0}^{2}}\right)^{1 / 2}$ is calculated to be $9.7 \mathrm{~nm}$. This value indicates the geometrical scale of the chain, although the interaction between polymers and the larger diameter of a fluorine atom than a hydrogen atom enlarges the distance actually. The charging state of a cluster of this size can be estimated crudely by a charge balance equation based on the orbitalmotion-limited probe theory ${ }^{26}$ and plasma neutralization condition. ${ }^{27}$ The calculation predicts that a cluster is charged up with a few electrons in a typical plasma condition, where the plasma density, the electron temperature, and the ion temperature are assumed to be $10^{9} \mathrm{~cm}^{-3}, 3 \mathrm{eV}$, and $0.03 \mathrm{eV}$, respectively. ${ }^{28}$ It is also assumed that the number density of the clusters is less than a half of the plasma density. Since the charge of a cluster increases with its size, a larger cluster tends to be trapped more easily in the plasma. The trapped clusters continue to grow up by the mutual aggregation and by deposition of film precursor around the clusters to particles with micrometer sizes until they are separated with each other by the Coulomb repulsive force. ${ }^{29}$

\section{CONCLUDING REMARKS}

By the GPC measurement, it has been verified that polymerized species with molecular weights up to 2000 are contained in the deposited film, and further polymerized species with molecular weights around 100000 are contained in particles grown up in the gas phase. This suggests that the polymerization up to 2000 molecular weights proceeds frequently in the gas phase. These products are transported to the substrate surface and taken into the crosslinking reactions with the assistance of ion bombardments. On the other hand, those remained in the plasma continue to grow by mutual aggregation with more effective ion bombardments up to 100000 molecular weights and tend to be trapped by the electron charging. The aggregation between these largely polymerized species leads to the formation of micrometer sized particles.

It is found by the XPS measurement that the F/C atomic ratio of the reaction products as both thin films and solid particles is reduced markedly in a comparison with that of the source monomer. This indicates that a $\mathrm{C}-\mathrm{F}$ bond is weaker than a $\mathrm{C}-\mathrm{C}$ bond, and a fluorine atom is more likely to be taken off than a carbon atom from the product by electron, radical, or ion collision in the gas phase. The activated site of the monomer and the oligomer by the fluorine removal enhances the formation of the highly branched and crosslinked molecules. In particular, in the solid particle formation, its precursor stays in the gas phase for a longer time than in the case of film formation. Therefore, the detachment 
of fluorine atoms progresses more likely in the molecule composing the particle due to more frequent bombardment of reactive species. Consequently, the particles include more carbon atoms than the thin film deposited on the surface.

A part of polymerized molecules produced in the gas phase reach to the surface and the rest continue polymerization while staying in the gas phase. On the surface, the polymerized molecules construct crosslinked network in the film through the activation process induced by ion irradiation from the plasma. Thus, the film is composed of the polymer network as well as smaller unlinked species like monomer, oligomer, etc. On the other hand, the molecules staying in the gas phase become ultralarge polymers with the molecular weight of about 100000 . These are trapped by charging with electrons in the plasma, leading to the rapid growth of solid particles.

Besides the polymerization mechanisms, it is suggested from the present experiment that the porous structure of the THF washed film prepared at higher pressure may be interesting for an application to low-dielectric constant material. Therefore, the microscopic structure is under investigation.

\section{ACKNOWLEDGMENTS}

The present work was supported in part by a Grant-inAid for scientific research from the Ministry of Education, Science, Sport, and Culture, and a grant for young researchers from Kyoto University Venture Business Laboratory (KU-VBL). The authors would like to thank Professor Shinzaburo Ito (Department of Polymer Chemistry, Kyoto University), Dr. Michiaki Mabuchi (KU-VBL), and Dr. Kenji Ishida (KU-VBL) for the GPC measurement and the significant discussion. Thanks are also due to Yuzuru Shimazaki and Yuji Sakurai for technical supports of GPC and Atsushi Itoh for experimental assistance. One of the authors (K.T.) is grateful for Research Fellowships of the Japan Society for the Promotion of Science for Young Scientists (Code 03323).
${ }^{1}$ R. A. H. Heinecke, Solid-State Electron. 18, 1146 (1975).

${ }^{2}$ J. L. Vossen, J. Electrochem. Soc. 126, 319 (1979).

${ }^{3}$ J. W. Coburn, J. Vac. Sci. Technol. A 12, 617 (1994).

${ }^{4}$ K. Endo and T. Tatsumi, Appl. Phys. Lett. 68, 2864 (1996).

${ }^{5}$ M. Inayoshi, M. Ito, M. Hori, T. Goto, and M. Hiramatsu, J. Vac. Sci. Technol. A 16, 232 (1998).

${ }^{6}$ K. Takahashi, M. Hori, M. Inayoshi, and T. Goto, Jpn. J. Appl. Phys., Part 1 35, 3635 (1996)

${ }^{7}$ K. Takahashi, M. Hori, and T. Goto, J. Vac. Sci. Technol. A 14, 2011 (1996)

${ }^{8}$ K. Miyata, M. Hori, and T. Goto, J. Vac. Sci. Technol. A 15, 568 (1997).

${ }^{9}$ H. Sugai and H. Toyoda, J. Vac. Sci. Technol. A 10, 1193 (1992).

${ }^{10}$ D. Vender, M. Haverlag, and G. S. Oehrlein, Appl. Phys. Lett. 61, 3136 (1992).

${ }^{11}$ J. P. Booth, Plasma Sources Sci. Technol. 8, 249 (1999).

${ }^{12}$ J. P. Booth and G. Cunge, J. Plasma Fusion Res. 75, 821 (1999).

${ }^{13}$ C. Suzuki, K. Sasaki, and K. Kadota, J. Vac. Sci. Technol. A 16, 2222 (1998).

${ }^{14}$ E. Stoffels, W. W. Stoffels, and K. Tachibana, Rev. Sci. Instrum. 69, 116 (1998).

${ }^{15}$ W. W. Stoffels, E. Stoffels, and K. Tachibana, J. Vac. Sci. Technol. A 16, 87 (1998).

${ }^{16}$ S. Imai and K. Tachibana, Jpn. J. Appl. Phys., Part 2 38, L888 (1999).

${ }^{17}$ B. Bartlett, L. J. Buckley, and D. J. Godbey, J. Vac. Sci. Technol. B 17, 90 (1999).

${ }^{18}$ CRC Handbook of Chemistry and Physics, 77th ed. (CRC, Boca Raton, FL, 1996).

${ }^{19}$ M. M. Millard, J. J. Windle, and A. E. Pavlath, J. Appl. Polym. Sci. 17, 2505 (1973)

${ }^{20}$ K. Hozumi, K. Kitamura, and T. Kitade, Bull. Chem. Soc. Jpn. 54, 1392 (1981).

${ }^{21} \mathrm{~K}$. Takahashi and K. Tachibana, Proceedings of the 21st Symposium Dry Process, Tokyo (IEE, Tokyo, 1999), p. 15.

${ }^{22}$ The Sigma-Aldrich Library of Chemical Safety Data, Sigma-Aldrich Corp., 1985.

${ }^{23}$ D. T. Clark and D. Shuttleworth, J. Polym. Sci., Polym. Chem. Ed. 18, 27 (1980)

${ }^{24}$ M. S. Barnes, J. H. Keller, J. C. Forster, J. A. O'Neill, and D. K. Coultas, Phys. Rev. Lett. 68, 313 (1992).

${ }^{25}$ P. J. Flory, Principles of Polymer Chemistry (Cornell University Press, New York, 1953)

${ }^{26}$ L. Schott, Plasma Diagnostics, edited by W. Lochte-Holtgreven (Wiley, New York, 1970), Chap. 11

${ }^{27}$ J. P. Boueuf and C. Punset, Dusty Plasmas, Physics, Chemistry and Technological Impacts in Plasma Processing, edited by A. Bouchoule (Wiley, England, 1999), Chap. 1, p. 50.

${ }^{28}$ M. A. Lieberman and A. J. Lichtenberg, Principles of Plasma Discharges and Materials Processing (Wiley, New York, 1994).

${ }^{29}$ M. Shiratani, H. Kawasaki, T. Fukuzawa, T. Yoshioka, Y. Ueda, S. Singh, and Y. Watanabe, J. Appl. Phys. 79, 104 (1996). 